\title{
Aerodynamic Optimization of a High-Lift System with Kinematic Constraints
}

Dirk M. Franke

\begin{abstract}
The design of the high-lift system is crucial for the economical success of a commercial airplane. The aim of this work is to find an optimized high-lift system with improved aerodynamic performance and kinematic properties. The analysis presented in this work covers the simulation of the aerodynamics and the kinematics, which are embedded in an optimization framework. The optimization revealed a configuration that fulfills the kinematic constraints and an improvement in the aerodynamic performance. It can be stated that the consideration of additional kinematic constraints for an aerodynamic setting optimization leads to a more realistic design.
\end{abstract}

\section{Introduction}

Commercial aircrafts use high-lift systems at low speeds during take-off and landing to generate sufficient lift. Although high-lift systems are used only for a short time during the complete flight their influence on the cost efficiency of the aircraft is significant [3]. To withstand in the international market it is therefore essential to design a high-lift system with a high performance. High performance encourages e.g. the designer to realize low approach speeds by a high aerodynamic performance, i.e. high lift coefficients.

The understanding of the aerodynamics of multi-element airfoils goes back to the work of A.M.O. Smith [10] in 1975. This fundamental work presents in detail the mechanisms that trigger the aerodynamics of high-lift devices w.r.t. gaps. Smith set up five primary effects of gaps that dominate the high-lift aerodynamics, namely slat effect, circulation effect, dumping effect, off-the-surface pressure recovery and fresh-boundary-layer effect. Nowadays a focus in the field of high-lift research deals with multidisciplinary aspects as shown by van Dam et al. [4], more recently by

Dirk M. Franke

German Aerospace Center, Institute of Aerodynamics and Flow Technology, Lilienthalplatz 7, 38108 Braunschweig, Germany, e-mail: d.franke@dlr.de 
Takenaka et al. [11] and Kolla et al. [7]. Van Dam optimized the aero-mechanical system while analyzing the trailing edge kinematics and taking the aerodynamic performance of the flap setting from a database. Takenaka investigated the trade-off between aerodynamics and kinematics with a genetic algorithm in take-off configuration. Kolla et al. concentrate on aerodynamic shape optimization of the flap in landing configuration while fulfilling a stowability constraint.

The following work covers the aero-mechanical optimization of a three element high-lift airfoil. The high-lift system is provided with a circular arc track for the slat and a track-linkage system for the flap. Further on, the impact of the kinematics boundary condition on the final configuration is investigated. Therefore the same test case is optimized also in a monodisciplinary (purely aerodynamic) manner.

The aim of this work is to design a high-lift system with improved aerodynamic performance (setting optimization) and a realizable track-linkage mechanism.

\section{Methods}

Figure 1 shows the aero-mechanical process chain that is embedded in the optimization environment CHAeOPS [12]. For the purely aerodynamic optimization the kinematics module is neglected and the set of parameters is changed.

Test Case: As test case the geometry of the three-element airfoil L1T2 is taken [8]. The airfoil has a slat with a chord length of $c_{\text {Slat }}=12.5 \% \mathrm{c}$ and a flap with a chord length of $c_{\text {Flap }}=33 \% c$ ( $c$ : chord length of the clean airfoil). In contrast to Moir [8] the geometry of the cove close to the lower side of the main element was changed. The shape of the cove does not collapse with the shape of the flap from its leading edge to the lower trailing edge of the main element.

From the kinematics perspective a circular arc track for the slat and a track-linkage system for the flap is regarded. Since the current work concentrates on the flap mechanism, the slat arc track radius is fixed and only the deflection angle is adjusted. The free stream conditions for the test case read: $\mathrm{Ma}=0.197, \mathrm{Re}=3.52 \mathrm{e}+6, \alpha=20.18^{\circ}$.

Parameterization: For the aero-mechanical optimization, 16 parameters are defined (see figure 1):

- coordinates of the nodes of the trailing edge mechanism $\left(\mathrm{P}_{x}, \mathrm{P}_{z}, \ldots, \mathrm{P} 6_{x}, \mathrm{P} 6_{z}\right)(14$ parameters)

- angle at the actuator $\phi$ (1 parameter)

- slat deflection angle $\delta_{\text {Slat }}(1$ parameter)

The common aerodynamic parameters, namely gap, overlap and deflection angle for the flap are defined indirectly by the parameters that define the geometry of the mechanism.

For the monodisciplinary optimization instead, the four parameters are directly applied which define:

- gap, overlap and deflection angle $\delta_{\text {Flap }}$ of the flap (3 parameters)

- slat deflection angle $\delta_{\text {Slat }}(1$ parameter) 
Optimization Algorithm: The optimization is performed with the SUBPLEX algorithm which is a deterministic, gradient free optimization algorithm developed by Rowan. According to Rowan [9] "the subplex method's approach is to decompose the problem into low-dimensional subspaces that the Simplex method can search efficiently."

As shown by Wild [12], the SUBPLEX algorithm is suitable for high-lift optimization. The algorithm is characterized by a good handling of noisy functions and by a well convergence behavior against strongly different sensitivities of the parameters. Kinematics Module: The kinematics analysis is done by a tool developed by the author. The discrete positions of the mechanism are computed by a numerical approach. The geometry of the mechanism is described by the formulation of vector loop equations. These equations are solved iteratively by a Newton-Raphson method. Based on the discrete positions of the mechanism the flap position is computed and therewith the gap, overlap and deflection angle for each position is evaluated. The outputs of the kinematics module are the discrete position of the flap depending on the actuator angle $\phi$ and a scalar value that indicates a collision between the main element and the flap during the deployment. A collision is only detected when it takes place in front of the spoilers. The validation of the kinematics module was done by a comparison of the trajectory path of the flap with a kinematics tool for trailing edge kinematics TEFlaMeS [6]. TEFlaMeS uses an analytical method to compute the trajectory path of the flap. The results show a very good accordance. Computational Mesh: A high mesh quality is important to resolve the complex flow phenomena around a three-element airfoil. In this study a focus of the meshing process is laid on the smoothness of the mesh, boundary layer and wake resolution for changing flap and slat settings. The meshes are always generated from scratch. The computational meshes are generated with the mesh generator MegaCADs [1]. The topology of the mesh is constant in the near wall and wake region where the physical domain is discretized with structured elements. In the outer field unstructured mesh elements are used, and so the number of elements changes.

Flow Solver: For the prediction of the integral coefficients the DLR-TAU code is used [5]. The code is a finite-volume CFD (computational fluid dynamics) solver for unstructured meshes. The code solves the compressible, three-dimensional, unsteady Reynolds averaged Navier-Stokes (RANS) equations. In this work the oneequation turbulence model from Spalart Allmaras with Edwards modifictaion (SAE) [2] in fully turbulent mode is used.

With the mentioned solver settings and the described meshing approach the standard L1T2 test case as presented by Moir [8] was computed. The results are in good agreement with the experimental data [8] and numerical results [12].

Optimization problem: The objective of the aero-mechanical optimization problem is the maximization of the lift coefficient at a fixed angle of attack while satisfying kinematic and an aerodynamic constraints. The kinematic constraint implies a collision-free trajectory path of the flap. The aerodynamic constraint is applied to control the pitching moment of the configuration and is realized with a penalty function. This constraint is imposed to ensure that the gain in the lift coefficient is not achieved by increasing the load on the aft of the main element and the flap (higher 
pitch down moment). This would result in a probable necessary down force to trim the configuration and consequently in an overall reduction of the lift coefficient. For the aero-mechanical optimization the flow around a configuration is only evaluated if the kinematic analysis reveals a collision-free trajectory path of the flap. The only change in the objective function for the monodisciplinary optimization is done by neglecting the kinematic constraint.

\section{Results}

The mono- and dualdisciplinary optimizations reveal an improvement in the aerodynamic performance while fulfilling its constraints. The optimized configurations show strong deviations in the flap setting and hence in its pressure coefficient distribution and its aerodynamic coefficients compared with the initial configuration. The major difference between the final configurations is the gap size of the flap.

Aerodynamics: Both final configurations show an improvement in the lift coefficient for the design point. An improvement of $4.2 \%$ for the aero and $1.8 \%$ for the aero-mechanical optimized configuration is obtained. The increase in the lift coefficient is mainly originated on the main element for both optimized configurations and on the flap for the aero-mechanical optimized configuration. Now we consider the pressure coefficient distribution (figure 2 a). On the flap we recognize a strong suction peak increase for the aero-mechanical optimized configuration. On the main element an increase of the suction peak is recognizable for both optimized configurations. In the cove region of the main element the aero-mechanical optimized configuration shows a lower pressure level, hence the flow has a higher velocity. On the suction side of the main element the aero optimized configuration shows a slightly lower pressure coefficient level. On the slat, a reduction of the suction peak, higher pressure level on the suction side close to the aft and lower velocities in the cove region (higher pressure coefficient) are perceived for the final configurations. According to figure $2 \mathrm{~b}$, the lift coefficient for the design point $\left(\alpha=20.18^{\circ}\right)$, the maximum lift coefficient $\left(C_{\mathrm{L}, \max }\right)$ and the angle of attack for $C_{\mathrm{L}, \max }$ are increased for both final configurations. In the linear region of the functional $C_{L}(\alpha)$ the aeromechanical optimized configuration shows lower values for the lift coefficient than the initial and the aero-optimized configuration.

Figure 3 shows the results concerning the Kinematics. On the left side we observe how the size of the gap, the overlap and the deflection angle of the flap varies via the trajectory path of the flap. The final, optimized flap setting is observed at about $88 \%$ of the trajectory of the flap. First of all we can recognize that there is a positive gap size for the whole trajectory between the trailing edge of the main element and the flap. In retracted position the gap size is positive which is based on the geometry of the airfoil. Furthermore the flap deflection angle shows small negative values at the beginning of the deployment. The overlap is constantly decreasing during the deployment.

Figure 3 right shows the initial and final mechanisms with retracted and deployed 
flap. The upper figure shows the main changes in the final mechanism that impact the trajectory of the flap. These are a higher angle of the track $\alpha_{\text {Track }}$ and an almost vertical adjusted rear link. For the final mechanism with deployed flap it is obvious that the gap size and the overlap for the final configuration has increased strongly (table 1).

The Optimization needed 148 loops for the aero and 433 loops for the aeromechanical approach to achieve the convergence criteria. About $16 \%$ of the optimization loops of the aero-mechanical approach were performed without evaluating CFD due to the unfulfilled kinematic constraints. For the aero optimization $3 \%$ of the loops were not evaluated with CFD.

\section{Discussion}

Aerodynamics: The lift coefficient of the slat is decreased for both final configurations due to an increase of the deflection angle. This leads to a decreased suction peak but also to an increase in the area that is surrounded by the pressure coefficient distribution close to the aft of the slat. On the main element the increase in the lift coefficient is gained in an increase of the suction peak (less dominant slat effect) and for the aero optimized configuration in a slightly lower pressure level on the suction side due to the smaller gap size (more dominant circulation effect). In the cove region of the main element the aero-mechanical optimized configuration has increased velocities which originate from higher velocities in the region of the leading edge of the flap (dumping effect). The flap shows only for the aero-mechanical optimized configuration an increased lift coefficient due to the increased gap size. This results in a less dominant slat effect (main element to flap), therewith the suction peak rises.

According to the $C_{L}-\alpha$ curve both optimized configurations show close to the design point and above an improved aerodynamic performance in comparison with the initial configuration. The fact that we obtain not only a higher performance at the design point but also an increased $C_{\mathrm{L}, \max }$ is favorable.

Kinematics: By the aero-mechanical optimization we obtain a mechanism that fulfills the requirements of the kinematic constraints. However, when looking at the final mechanism we can notice that the bars P0P1, P1P2 may collide with the rear spar of the main element. This is unfavorable but may be avoided by using a linear actuator instead of the rotational actuator. These bars affect the trajectory path of the flap only by limiting the maximum deployable position. A possibility to increase the collision-free domain of the mechanism is to redesign the cove of the main element that does not necessarily collapse with the shape of the flap.

The Optimization problem limits the domain in which the possible best solution is located by evaluating the aerodynamic coefficients at a fixed angle of attack. By this approach we sort out in advance configurations that have firstly their $C_{\mathrm{L}, \max }$ at a lower angle of attack than the design angle of attack $\left(\alpha=20.18^{\circ}\right)$ and secondly a lower $C_{L}$ at the design point, although these configurations may have a higher 
$C_{\mathrm{L}, \max }$.

The Parameterization has a crucial influence on the optimization result. Here we investigate the influence of the parameterization, i.e. if the deviations in the final settings are based on the parameterization. An optimization was performed to find a collision-free flap mechanism that reaches the aerodynamic parameters (gap, overlap, deflection angle) of the aero optimized configuration. This optimization revealed either a collision-free system but strong deviations in the target aerodynamic parameters or a system with matching aerodynamic parameters but a collision. As a consequence a different type of mechanism need to be designed to realize the aero optimized flap setting or the design problem has to be solved iteratively.

\section{Conclusions}

The objective of the present work to design a high-lift system with improved aerodynamic performance and with a realizable track-linkage mechanism was successfully achieved. By considering the design task as a sequence of optimizations, firstly aerodynamic setting optimization, secondly optimization of the geometry of the mechanism, the design process becomes a cost intensive, iterative process. The results reveal that there is a great benefit in simulating not only the aerodynamics but also the kinematics to gain a more realistic design.

Future activities may be addressed to multi point optimizations of the take-off and landing configuration with a given type of mechanism. The optimization of $C_{L, \max }$ instead of $C_{L}$ at a given angle of attack would also be a valuable next step. However the cost of the evaluation of $C_{L, \max }$ is far more expensive.

\section{References}

1. Brodersen O., Ronzheimer A., Ziegler R., Kunert T., Wild J., Hepperle M. (1998) Aerodynamic Applications using MegaCads. 6th Intern. Conference on Numerical Grid Generation, Ed.: M.Cross et. al., London.

2. Edwards J.R., Chandra S. (1996) Comparison of Eddy Viscosity-Transport Turbulence Models for Three-Dimensional, Shock-Separated Flowfields. AIAA Journal, Vol.34, No.4: 756763.

3. van Dam C. P. (2002) The aerodynamic design of multi-element high-lift systems for transport airplanes. Progress in Aerospace Sciences, Vol. 38, No. 2: 101-144.

4. van Dam C. P., Shaw S. G., Vander Kam J. C., Rudolph P. K. C., Kinney D (1999) Aeromechanical design of high-lift systems. Aircraft Engineering and Aerospace Technology, Vol. 71, No. 5: 436-443.

5. Gerhold T. (2005) Overview of the Hybrid RANS Code TAU. MEGAFLOW - Numerical Flow Simulation for Aircraft Design. Notes on Numerical Fluid Mechanics and Multidisciplinary Design, Vol. 89.

6. Holert B.,Carl U. (2005) Rechnerunterstützte Synthese von Klappenführungsmechanismen, Project report, No.:04-158, v1.08, Hamburg University of Technology, Institute of Aircraft System Engineering. 
7. Kolla M. L., Yokata J. W., Lassaline J. V, Fejtek I. (2009) Stowability Constraint Within a Two-Dimensional Aerodynamic Optimization Method. Journal of Aircraft, Vol.46, No.2: 696-698.

8. Moir I. R. M. (1994) Measurement on a Two-Dimensional Airfoil with High-Lift Devices. AGARD AR 303: A2-1-A2-12.

9. Rowan T. (1990) Functional Stability Analysis of Numerical Algorithms. Dissertation, Department of Computer Sciences, University of Texas at Austin, USA.

10. Smith A. M. O. (1975) High-Lift Aerodynamics. Journal of Aircraft, Vol.12, No.6: 501-530.

11. Takenaka K., Nakahashi K. (2008) Aerodynamic optimization of 2-D High-Lift Device under Kinematic Constraints. 5th European Congress on Computational Methods in Applied Sciences and Engineering (ECCOMAS 2008), Venice, Italy.

12. Wild J. (2001) Numerische Optimierung von zweidiminsionalen Hochauftriebskonfigurationen durch Lösung der Navier-Stokes-Gleichungen. Research Report 2001-11. DLR, Institute of Aerodynamics and Flow Technology.

Table 1 Slat and flap setting for initial and final configurations

\begin{tabular}{lllll}
\hline & Slat & Flap & \\
& $\phi_{\text {Slat }}\left[{ }^{\circ}\right]$ & $\phi_{\text {Flap }}\left[{ }^{\circ}\right]$ & GapFlap $/ \mathrm{c}[\%]$ & Overlap ${ }_{\text {Flap }} / \mathrm{c}[\%]$ \\
\hline initial & 20.00 & 22.56 & 2.36 & 2.49 \\
final: aero & 25.19 & 22.37 & 1.52 & 3.80 \\
final: aero-mechanical & 25.08 & 20.31 & 3.86 & 4.40 \\
\hline
\end{tabular}

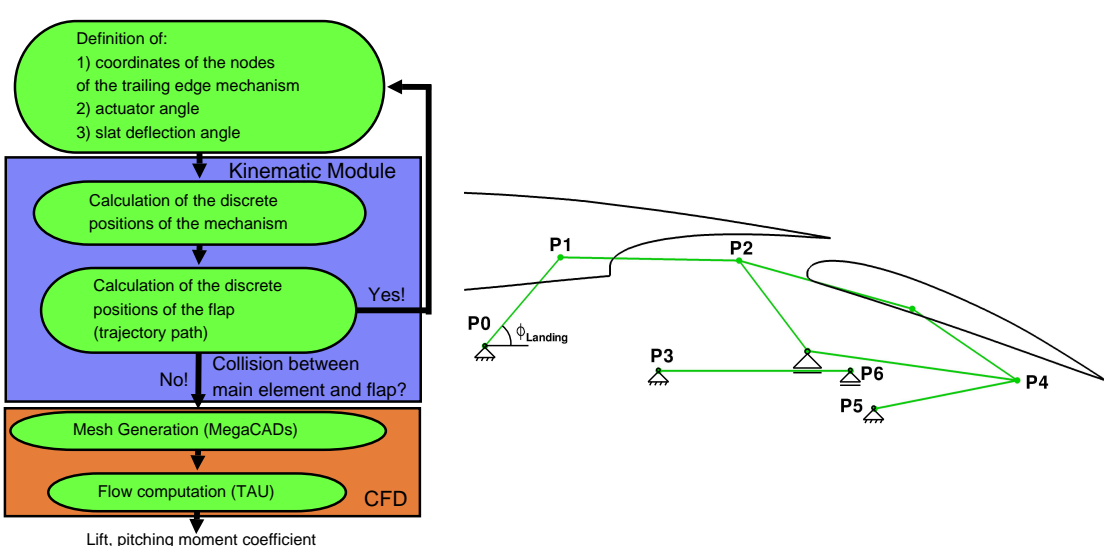

Fig. 1 Left: Process chain of coupled aero-mechanical analysis; right: Parameters of the aeromechanical optimization 


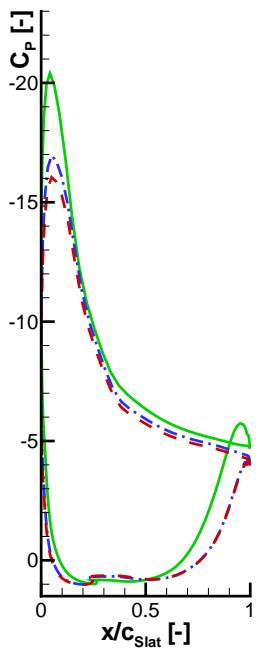

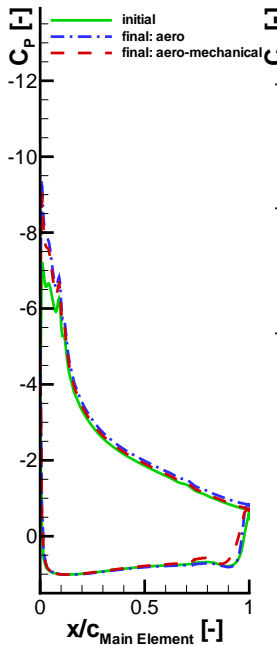

a)

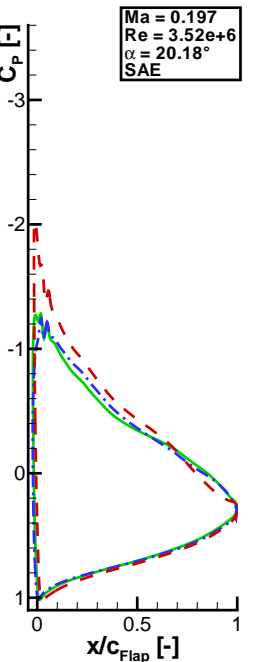

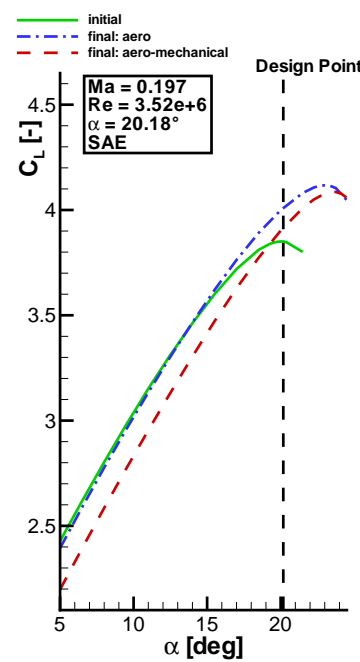

Fig. 2 a) Pressure coefficient distribution for initial and optimized configurations; b) $C_{L^{-}} \alpha$ curve for initial and final configurations

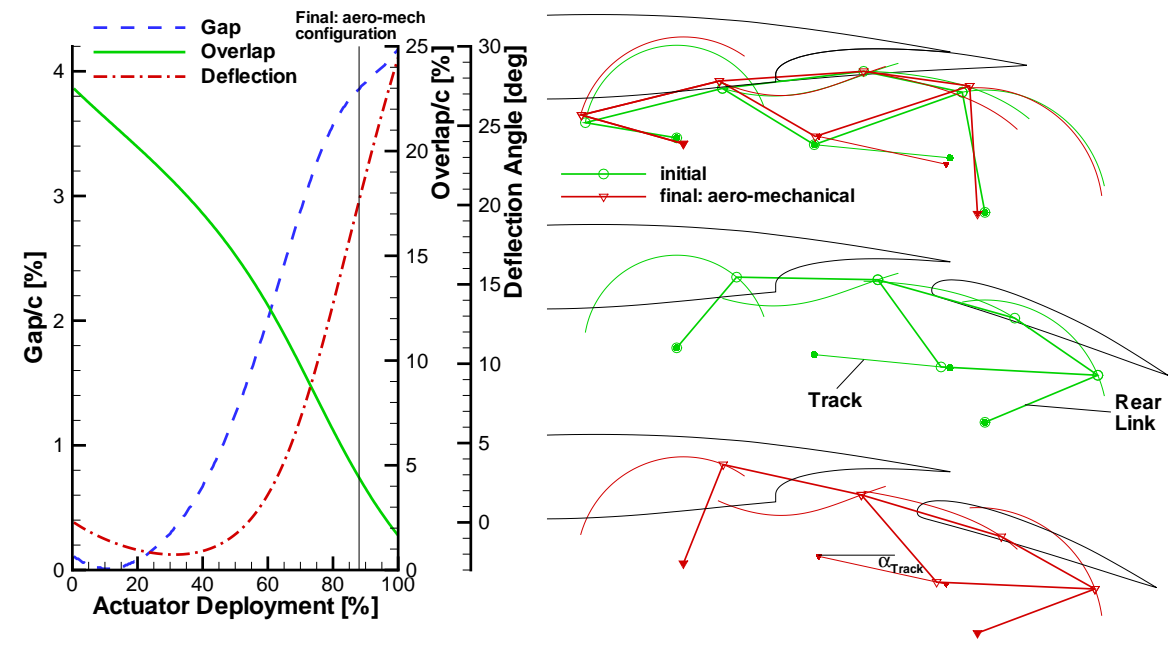

Fig. 3 Left: Gap, overlap and deflection angle of the flap via the trajectory path for final mechanism; right: Initial and final track-linkage mechanism with retracted and deployed flap 